\title{
REGULAR SELF-SIMILAR SOLUTIONS OF THE NONLINEAR HEAT EQUATION WITH INITIAL DATA ABOVE THE SINGULAR STEADY STATE
}

\author{
Philippe SOUPLET ${ }^{\mathrm{a}, \mathrm{b}}$, Fred B. WEISSLER ${ }^{\mathrm{c}, *}$ \\ ${ }^{a}$ Département de mathématiques, INSSET, Université de Picardie, 02109 St-Quentin, France \\ ${ }^{\mathrm{b}}$ Laboratoire de mathématiques appliquées, UMR CNRS 7641, Université de Versailles, \\ 45, avenue des Etats-Unis, 78035 Versailles, France \\ ${ }^{\mathrm{c}}$ LAGA, UMR CNRS 7539, Institut Galilée, Université Paris-Nord, 99, avenue J.-B. Clément, \\ 93430 Villetaneuse, France
}

Received 7 March 2001

ABSTRACT. - We prove the existence of positive regular solutions of the Cauchy problem for the nonlinear heat equation $u_{t}=\Delta u+|u|^{\alpha} u$, with initial value $\mu V$, for all $\mu>1$ close enough to 1 , where $V$ is the singular stationary solution in $\mathbb{R}^{N}$. This result is obtained when $N>2$ and $\frac{2}{N-2}<\alpha<\alpha^{*}$, where $\alpha^{*}$ is the critical power for the intersection properties of $V$ with regular stationary solutions. Moreover, for $\mu$ as above, there exist at least two positive regular solutions with initial value $\mu V$. These results are optimal since it is known that no such solution exists if $\alpha \geqslant \alpha^{*}$.

(C) 2003 L'Association Publications de l'Institut Henri Poincaré. Published by Elsevier B.V. All rights reserved

MSC: $35 \mathrm{~K} 15 ; 35 \mathrm{~K} 55$

RÉSUMÉ. - Nous montrons l'existence de solutions positives régulières du problème de Cauchy pour l'équation de la chaleur non linéaire $u_{t}=\Delta u+|u|^{\alpha} u$, avec donnée initiale $\mu V$, pour tout $\mu>1$ assez proche de 1 , où $V$ est la solution stationnaire singulière dans $\mathbb{R}^{N}$. Ce résultat est obtenu pour $N>2$ et $\frac{2}{N-2}<\alpha<\alpha^{*}$, où $\alpha^{*}$ est la puissance critique pour les propriétés d'intersection de $V$ avec les solutions stationnaires régulières. De plus, pour $\mu$ comme ci-dessus, il existe au moins deux solutions positives régulières avec donnée initiale $\mu V$. Ces résultats sont optimaux, car on savait déjà que de telles solutions ne peuvent exister si $\alpha \geqslant \alpha^{*}$.

(C) 2003 L'Association Publications de l'Institut Henri Poincaré. Published by Elsevier B.V. All rights reserved

\section{Introduction}

In this paper we study solutions of the nonlinear heat equation

$$
u_{t}=\Delta u+|u|^{\alpha} u, \quad t>0, x \in \mathbb{R}^{N},
$$

\footnotetext{
* Corresponding author.

E-mail addresses: souplet@math.uvsq.fr (P. Souplet), weissler@math.univ-paris13.fr (F.B. Weissler).
} 
which have singular initial values. Here $u=u(t, x) \in \mathbb{R}, \alpha>0$ and we sometimes write $u(t)$ for the spatial function $u(t, \cdot)$. If $N>2$ and $\alpha>\frac{2}{N-2}$, then (1.1) has a positive singular stationary solution $V(x)$ given by

$$
V(x)=\beta^{1 / \alpha}|x|^{-2 / \alpha},
$$

where

$$
\beta=\frac{2}{\alpha}\left(N-2-\frac{2}{\alpha}\right) .
$$

$V$ is a stationary solution of (1.1) in the sense of tempered distributions: each term of $(1.1)$ with $u(t, x)=V(x)$ is in $L^{1}\left(\mathbb{R}^{N}\right)+L^{\infty}\left(\mathbb{R}^{N}\right)$. Even though $V$ is a stationary solution, it turns out, for certain values of $\alpha$, that $V$ is not the minimal positive solution of (1.1) whose initial value is $V$. Indeed, if $N>10$, let

$$
\alpha^{*}=\frac{4}{N-4-2 \sqrt{N-1}} .
$$

(We take $\alpha^{*}=\infty$ if $N \leqslant 10$.) This number first appeared in [5] in the study of stationary solutions of (1.1). Galaktionov and Vazquez [3] have proved that if $\frac{2}{N-2}<\alpha<\alpha^{*}$, then there exists a $C^{\infty}$, positive solution $u(t, x)$ of (1.1), for $t>0$, such that $u(t) \rightarrow V$ in $\mathcal{S}^{\prime}$, indeed in $L^{1}\left(\mathbb{R}^{N}\right)+L^{\infty}\left(\mathbb{R}^{N}\right)$, as $t \rightarrow 0+$.

The purpose of this paper is to improve the result of Galaktionov and Vazquez by showing that initial values $\mu V$ are allowed for some $\mu>1$.

THEOREM 1.1. - Let $N>2$ and $\frac{2}{N-2}<\alpha<\alpha^{*}$. There exists $\varepsilon>0$ such that for all $\mu \in(1,1+\varepsilon)$, there is a $C^{\infty}$ positive solution $u$ of $(1.1)$ such that

$$
u(t) \rightarrow \mu V \quad \text { in } L^{1}\left(\mathbb{R}^{N}\right)+L^{\infty}\left(\mathbb{R}^{N}\right) \text {, as } t \rightarrow 0+.
$$

Moreover, $u \in C\left(0, \infty ; L^{q}\left(\mathbb{R}^{N}\right)\right)$ for all $q>\frac{N \alpha}{2}$, and $\lim _{t \rightarrow \infty} u(t)=0$ in $L^{q}\left(\mathbb{R}^{N}\right)$ for all $q>\frac{N \alpha}{2}$.

This result calls for several remarks. First of all, the power $\alpha^{*}$ is optimal in Theorem 1.1. By Theorem 10.4 in [3], no nonnegative solution of (1.1) with intial value above $V$ exists if $\alpha \geqslant \alpha^{*}$. On the other hand, Theorem 10.4 in [3] also states that if $\frac{2}{N-2}<\alpha<\alpha^{*}$, then there is no solution of (1.1) with initial value above $V$ and which remains above $V$ for $t>0$. Theorem 1.1 shows that this cannot be improved. Also, it should be pointed out that if $\mu$ is sufficiently large, then there is no local nonnegative solution of (1.1) with initial value $\mu V$. See Theorem 1 in [9].

For the equation with $|u|^{\alpha} u$ replaced by $\mathrm{e}^{u}$, Vazquez [7] has shown that there exists a regular solution whose initial value is equal to the singular stationary solution plus a small positive constant.

Finally, the situation for singular stationary solutions contrasts markedly with that for initial values which are multiples of regular stationary solutions. Indeed, if $\phi$ is a positive regular stationary solution of (1.1) either on $\mathbb{R}^{N}$ or on a bounded domain (with Dirichlet boundary conditions), then the solution of (1.1) with initial value $\mu \phi$, for any $\mu>1$ is 
nondecreasing in time, and therefore exhibits one of the following three behaviors: finite time blow up, infinite time blow up, or convergence to another positive regular stationary solution.

We prove Theorem 1.1 by proving the existence of a positive, radially symmetric (forward) self-similar solution of (1.1) with the necessary properties. Such a solution is given by

$$
u(t, x)=t^{-1 / \alpha} f\left(\frac{r}{\sqrt{t}}\right),
$$

where $r=|x|$. The function $f:[0, \infty) \rightarrow \mathbb{R}$ is called the (spatial) profile of the solution $u$; indeed $u(1, x)=f(|x|)$. As is well-known, the function $u$ given by (1.5) is a solution of (1.1) if and only if $f$ is a solution of the profile equation:

$$
f^{\prime \prime}(r)+\left(\frac{N-1}{r}+\frac{r}{2}\right) f^{\prime}(r)+\frac{f(r)}{\alpha}+|f(r)|^{\alpha} f(r)=0 .
$$

The initial value problem associated with (1.6) was first studied in [4]. In particular (Theorem $5^{\prime}$ in [4]), if $f_{\lambda}$ is the solution of (1.6) such that $f_{\lambda}(0)=\lambda$ and $f_{\lambda}^{\prime}(0)=0$, then

$$
L(\lambda) \equiv \lim _{r \rightarrow \infty} r^{2 / \alpha} f_{\lambda}(r)
$$

exists and is a locally Lipschitz function of $\lambda \in \mathbb{R}$. If $u(t, x)$ is the resulting solution of (1.1) given by (1.5) with $f=f_{\lambda}$, then it is easy to see that $|u(t, x)| \leqslant M|x|^{-2 / \alpha}$ (for some $M>0)$ and $u(t, x) \rightarrow L(\lambda)|x|^{-2 / \alpha}$, uniformly on any subset of $\mathbb{R}^{N}$ bounded away from 0 , and so $u(t) \rightarrow L(\lambda)|x|^{-2 / \alpha}$ in $L^{1}\left(\mathbb{R}^{N}\right)+L^{\infty}\left(\mathbb{R}^{N}\right)$, as $t \rightarrow 0+$. It is also easy to check that $u(t)$ satisfies the integral equation associated to $(1.1)$ with $u(0)=L(\lambda)|x|^{-2 / \alpha}$.

The point of [4] was to show that if $\frac{2}{N}<\alpha<\frac{4}{N-2}$, then $L\left(\lambda_{0}\right)=0$ is attained for some $\lambda_{0}>0$ such that $f_{\lambda_{0}}(r)>0, \forall r>0$. Moreover, the analysis in [4] shows (for these values of $\alpha$ ) that there exist infinitely many pairs $\lambda_{1}, \lambda_{2}$ such that $0<\lambda_{1}<\lambda_{2}<\lambda_{0}, L\left(\lambda_{1}\right)=$ $L\left(\lambda_{2}\right)$, and both $f_{\lambda_{1}}$ and $f_{\lambda_{2}}$ are everywhere positive. Indeed, the $\lambda_{0}$ shown to exist in the proof of Proposition 3.7 in [4] has the property that if $0<\lambda<\lambda_{0}$, then $f_{\lambda}(r)>0, \forall r>0$. Thus, $L$ is a nonnegative, continuous function on $\left[0, \lambda_{0}\right]$ with $L(0)=0$ and $L\left(\lambda_{0}\right)=0$; and so $L$ attains every value in the interval $\left(0, \max _{\lambda \in\left[0, \lambda_{0}\right]} L(\lambda)\right)$ at least twice. In other words, for these values of $L(\lambda)$, there are at least two positive, regular, self-similar solutions of (1.1) with the same singular initial value $L\left(\lambda_{1}\right)|x|^{-2 / \alpha}=L\left(\lambda_{2}\right)|x|^{-2 / \alpha}$. Furthermore, by Proposition 3.6 in [4], $L(\lambda)>0$ if $\lambda>0$ is sufficiently small, and so $\max _{\lambda \in\left[0, \lambda_{0}\right]} L(\lambda)>0$.

The point of the present paper is to show for $\alpha$ in the range specified in Theorem 1, that $L\left(\lambda^{\prime}\right)=\mu \beta^{1 / \alpha}$ is attained in (1.7) for some $\lambda^{\prime}>0$ such that $f_{\lambda^{\prime}}(r)>0, \forall r>0$ and for some value of $\mu>1$. In the subcritical case, $\alpha<\frac{4}{N-2}$, Theorem 1.1 is a direct consequence of Theorem 4.4 and Proposition 3.5 proved in the main body of this paper. Moreover, Dohmen and Hirose [2, Theorem 1.2] have shown that if $\frac{2}{N-2} \leqslant \alpha<\frac{4}{N-2}$ and $\lambda>\lambda_{0}$, then $f_{\lambda}$ assumes negative values, and so it must be that $\lambda^{\prime} \in\left(0, \lambda_{0}\right)$. (In fact, Dohmen and Hirose [2] show the uniqueness of the $\lambda_{0}$ proved to exist in [4]. See Weissler [8] for the same result in the case $N=1$ and Yanagida [10] in the case $\alpha \leqslant \frac{2}{N-2}$.) Since as just noted, if $\frac{2}{N}<\alpha<\frac{4}{N-2}$, then $L$ attains every value in the 
interval $\left(0, \max _{\lambda \in\left[0, \lambda_{0}\right]} L(\lambda)\right)$ at least twice, Theorem 1.1 has the following immediate, but unexpected, consequence.

THEOREM 1.2. - If $N>2$ and $\frac{2}{N-2}<\alpha<\frac{4}{N-2}$, then there exists $\varepsilon>0$ such that for all $\mu \in(0,1+\varepsilon)$, there are at least two different $C^{\infty}$ positive solutions $u$ of $(1.1)$ such that

$$
u(t) \rightarrow \mu V \quad \text { in } L^{1}\left(\mathbb{R}^{N}\right)+L^{\infty}\left(\mathbb{R}^{N}\right), \text { as } t \rightarrow 0+,
$$

$u \in C\left(0, \infty ; L^{q}\left(\mathbb{R}^{N}\right)\right)$ for all $q>\frac{N \alpha}{2}$, and $\lim _{t \rightarrow \infty} u(t)=0$ in $L^{q}\left(\mathbb{R}^{N}\right)$ for all $q>\frac{N \alpha}{2}$.

This result is all the more surprising since an existence and uniqueness result for solutions of the type described in Theorem 1.2 is already known. Specifically, Theorem 6.1 in [1], says that if $\alpha>\frac{2}{N}$ (with no upper limit) and if $\mu>0$ and $M>0$ are sufficiently small (and verify a certain nonlinear relationship), then there exists a unique (necessarily self-similar) solution $u$ of the integral equation corresponding to (1.1) with initial value $\mu|x|^{-2 / \alpha}$ such that

$$
\sup _{t>0} t^{\frac{1}{\alpha}-\frac{N}{2 q}}\|u(t)\|_{L^{q}} \leqslant M,
$$

where

$$
1<\frac{q}{\alpha+1}<\frac{N \alpha}{2}<q
$$

It is clear that the supremum in (1.8) is finite for the self-similar solutions which provide the non-unique solutions in Theorem 1.2. Nonetheless, for one of the two solutions, this supremum must stay bounded away from 0 , even as $\mu \rightarrow 0$.

The situation for critical and supercritical $\alpha$ is more intricate. In the supercritical case, we will show that $L(\lambda)$, which is known to be positive for $\lambda>0$, oscillates around $\beta^{1 / \alpha}$ infinitely often as $\lambda \rightarrow \infty$. In the critical case, $L(\lambda)=\beta^{1 / \alpha}$ for at least two values of $\lambda>0$. More precisely, we have the following results, which are proved in Section 4 below.

THEOREM 1.3. - Let $N>2$ and $\alpha=\frac{4}{N-2}$. There exists $\varepsilon>0$ such that for all $\mu \in[1,1+\varepsilon)$, there are at least two different $C^{\infty}$ positive solutions $u$ of $(1.1)$ such that

$$
u(t) \rightarrow \mu V \quad \text { in } L^{1}\left(\mathbb{R}^{N}\right)+L^{\infty}\left(\mathbb{R}^{N}\right) \text {, as } t \rightarrow 0+,
$$

$u \in C\left(0, \infty ; L^{q}\left(\mathbb{R}^{N}\right)\right)$ for all $q>\frac{N \alpha}{2}$, and $\lim _{t \rightarrow \infty} u(t)=0$ in $L^{q}\left(\mathbb{R}^{N}\right)$ for all $q>\frac{N \alpha}{2}$.

THEOREM 1.4. - Let $N>2$ and $\frac{4}{N-2}<\alpha<\alpha^{*}$. For every positive integer $m$, there exists $\varepsilon=\varepsilon(m)>0$ such that for all $\mu \in(1-\varepsilon(m), 1+\varepsilon(m))$, there are at least $\mathrm{m}$ different $C^{\infty}$ positive solutions $u$ of (1.1) such that

$$
u(t) \rightarrow \mu V \quad \text { in } L^{1}\left(\mathbb{R}^{N}\right)+L^{\infty}\left(\mathbb{R}^{N}\right), \text { as } t \rightarrow 0+,
$$

$u \in C\left(0, \infty ; L^{q}\left(\mathbb{R}^{N}\right)\right)$ for all $q>\frac{N \alpha}{2}$, and $\lim _{t \rightarrow \infty} u(t)=0$ in $L^{q}\left(\mathbb{R}^{N}\right)$ for all $q>\frac{N \alpha}{2}$. 
Our basic approach to studying the attainable values of $L(\lambda)$ is to transform (1.6) so that the behavior as $r \rightarrow \infty$ is translated into the behavior near 0 . Motivated by the precise asymptotic behavior of solutions to (1.6) as given in Theorem 1 in [6], we set

$$
f(r)=r^{-2 / \alpha} w\left(r^{-2}\right)=s^{1 / \alpha} w(s), \quad w(s)=s^{-1 / \alpha} f\left(\frac{1}{\sqrt{s}}\right)=r^{2 / \alpha} f(r),
$$

where $s=r^{-2}$. A straightforward calculation shows that $f:(0, \infty) \rightarrow \mathbb{R}$ satisfies (1.6) if and only if $w:(0, \infty) \rightarrow \mathbb{R}$ satisfies the following differential equation:

$$
\begin{aligned}
& 4 s^{2} w^{\prime \prime}(s)+4\left(\frac{2}{\alpha}-\frac{N-4}{2}\right) s w^{\prime}(s)-w^{\prime}(s) \\
& -\frac{2}{\alpha}\left(N-2-\frac{2}{\alpha}\right) w(s)+|w(s)|^{\alpha} w(s)=0 .
\end{aligned}
$$

We refer to (1.10) as the inverted profile equation, to reflect the fact that behavior at 0 and $\infty$ are interchanged in passing from $f$ to $w$. If $u$ is a self-similar solution of (1.1) with profile $f$, and if $f$ and $w$ are related by (1.9), then

$$
u(t, x)=r^{-2 / \alpha} w\left(\frac{t}{r^{2}}\right)
$$

This shows that $w$ is in fact the time profile of the self-similar solution, i.e. $u(t, 1)=$ $w(t)$, where by abuse of notation, we write $u(t, r)$ instead of $u(t, x)$ with $|x|=r$.

It is important to note that if $f$ and $w$ are related by (1.9), and if $w$ is continuous at $s=0$, then $\lim _{r \rightarrow \infty} r^{2 / \alpha} f(r)=w(0)$. Thus, studying solutions $w$ of (1.10) having a specified initial value $w(0)$ is equivalent to studying solutions $f$ of the original profile equation (1.6) with $\lim _{r \rightarrow \infty} r^{2 / \alpha} f(r)=w(0)$. If, in addition, $f$ is the profile of a self-similar solution $u$ of (1.1), then the initial value of this solution $u$ is given by $u(0, x)=w(0)|x|^{-2 / \alpha}$. Hence the study of solutions to (1.10) having a specified initial value $w(0)$ is related to the study of solutions to (1.1) with a specified singular initial value $u(0, x)=w(0)|x|^{-2 / \alpha}$.

Throughout this paper, $\beta, \alpha^{*}$ and $V$ are as in (1.3), (1.4) and (1.2) above. Moreover, we use the following notation:

$$
\gamma=\frac{2}{\alpha}-\frac{N-4}{2}
$$

For future reference, we note that:

$$
\begin{aligned}
& \beta>0 \quad \Leftrightarrow \quad \alpha>\frac{2}{N-2} \\
& \gamma>1 \quad \Leftrightarrow \quad \alpha<\frac{4}{N-2} .
\end{aligned}
$$

As is common practice, conditions such as $\alpha>\frac{2}{N-2}$ are meant to imply that $N>2$, and conditions such as $\alpha<\frac{4}{N-2}$ are meant to be vacuous if $N=1$ or 2 . Also, $g: \mathbb{R} \rightarrow \mathbb{R}$, and its primitive $G: \mathbb{R} \rightarrow \mathbb{R}$, are the functions given by: 


$$
g(x)=-\beta x+|x|^{\alpha} x, \quad G(x)=-\frac{\beta x^{2}}{2}+\frac{|x|^{\alpha+2}}{\alpha+2} .
$$

We define

$$
G_{\min }=\min _{x \in \mathbb{R}} G(x) .
$$

In the case $\alpha>\frac{2}{N-2}$, Eq. (1.10) has a unique nontrivial positive constant solution, i.e. $w(s) \equiv \beta^{1 / \alpha}$, which corresponds to the singular stationary solution $V$ of (1.1).

With the above notation, the inverted profile equation (1.10) takes the following form:

$$
4 s^{2} w^{\prime \prime}(s)+4 \gamma s w^{\prime}(s)-w^{\prime}(s)+g(w(s))=0 .
$$

Given a solution $w$ of (1.12), we define its "energy" by

$$
H(s)=H_{w, w^{\prime}}(s)=2 s^{2} w^{\prime}(s)^{2}+G(w(s)) .
$$

It is straightforward to check that

$$
H^{\prime}(s)=w^{\prime}(s)^{2}[1-4(\gamma-1) s]=w^{\prime}(s)^{2}\left[1-2 s\left(\frac{4}{\alpha}-(N-2)\right)\right]
$$

This last formula depends only on the fact that $G^{\prime}=g$ and Eq. (1.12), and not on the specific form of the functions $g$ and $G$.

The rest of this paper is organized as follows. In the next section, we prove local existence and uniqueness of solutions to the inverted profile equation (1.12) on an interval $[0, T]$. Because of the strong singularity at $s=0$, one cannot specify both $w(0)$ and $w^{\prime}(0)$. See Proposition 2.4 and Theorem 2.5 below. In the following section, we show that these solutions can be continued for all $s>0$ and investigate their asymptotic behavior as $s \rightarrow \infty$. In particular, Proposition 3.5 states that if $w$ is a solution of (1.12) such that $\lim _{s \rightarrow \infty} w(s)=0$, then the resulting self-similar solution of (1.1) obtained via (1.9) and (1.5) is regular. Finally, in the last section, we prove the main results of the paper. In the subcritical case, $\alpha<\frac{4}{N-2}$, we use a shooting argument based on the solutions $w$ of (1.12). See Theorem 4.4. In the supercritical and critical cases, we use properties of solutions to (1.12) near $s=0$ (Proposition 2.7) to obtain detailed information about the intersections of the solutions $f_{\lambda}$ of (1.6) with the singular solution $V$ and the values attained by $L(\lambda)$. See Lemmas $4.5-4.8$.

\section{Local existence and uniqueness theory for the inverted profile equation}

The purpose of this section is to prove an existence and uniqueness theorem for solutions of (1.12) on an interval $[0, T]$. To accomplish this, we need first to re-write (1.12) in various equivalent forms, and then transform it into an integral equation which has solutions continuous at $s=0$. To this end, we consider the three following equations:

$$
\frac{\mathrm{d}}{\mathrm{d} s}\left(4 s^{\gamma} \mathrm{e}^{\frac{1}{4 s}} w^{\prime}(s)\right)=-s^{\gamma-2} \mathrm{e}^{\frac{1}{4 s}} g(w(s))
$$




$$
\begin{aligned}
w^{\prime}(s)=s^{-\gamma} \mathrm{e}^{-\frac{1}{4 s}} & T^{\gamma} \mathrm{e}^{\frac{1}{4 T}} w^{\prime}(T)+\frac{1}{4} s^{-\gamma} \mathrm{e}^{-\frac{1}{4 s}} \int_{s}^{T} \sigma^{\gamma-2} \mathrm{e}^{\frac{1}{4 \sigma}} g(w(\sigma)) \mathrm{d} \sigma ; \\
w(t)= & w\left(t_{0}\right)+\left(T^{\gamma} \mathrm{e}^{\frac{1}{4 T}} \int_{t_{0}}^{t} s^{-\gamma} \mathrm{e}^{-\frac{1}{4 s}} \mathrm{~d} s\right) w^{\prime}(T) \\
& +\frac{1}{4} \int_{t_{0}}^{t} s^{-\gamma} \mathrm{e}^{-\frac{1}{4 s}} \int_{s}^{T} \sigma^{\gamma-2} \mathrm{e}^{\frac{1}{4 \sigma}} g(w(\sigma)) \mathrm{d} \sigma \mathrm{d} s .
\end{aligned}
$$

It is simple to verify that (2.1) is the same as (1.12). Moreover, $w$ is a solution of (2.1) on some interval $J \subset(0, \infty)$ if and only if $w$ is a solution of (2.2) on $J$, as long as $T \in J$. In a like manner, if $t_{0}$ is also in $J$, then $w$ is a solution of (2.3) on $J$ if and only if it is a solution of (2.2).

In order to study solutions which are continuous on $[0, \mathrm{~T}]$, we need the following elementary results.

LEMMA 2.1.-

$$
\lim _{s \rightarrow 0+} \frac{1}{4} s^{-\gamma} \mathrm{e}^{-\frac{1}{4 s}} \int_{s}^{T} \sigma^{\gamma-2} \mathrm{e}^{\frac{1}{4 \sigma}} \mathrm{d} \sigma=1 .
$$

COROLlaRY 2.2. - If $h:[0, T] \rightarrow \mathbb{R}$ is a continuous function, then

$$
\lim _{s \rightarrow 0+} \frac{1}{4} s^{-\gamma} \mathrm{e}^{-\frac{1}{4 s}} \int_{s}^{T} \sigma^{\gamma-2} \mathrm{e}^{\frac{1}{4 \sigma}} h(\sigma) \mathrm{d} \sigma=h(0) .
$$

LEMMA 2.3. -

$$
\lim _{T \rightarrow 0+} \frac{1}{4} T^{\gamma-2} \mathrm{e}^{\frac{1}{4 T}} \int_{0}^{T} s^{-\gamma} \mathrm{e}^{-\frac{1}{4 s}} \mathrm{~d} s=1 .
$$

Lemmas 2.1 and 2.3 are proved with l'Hôpital's rule. Corollary 2.2 is a straightforward consequence of Lemma 2.1.

Proposition 2.4. - If $w \in C^{2}((0, T])$ is a solution of $(2.1)$, then $w \in C^{1}([0, T])$. Moreover,

$$
w^{\prime}(0)=g(w(0))
$$

and $w$ is a solution of the following integral equation on $[0, T]$ :

$$
\begin{aligned}
w(t)= & w(0)+\left(T^{\gamma} \mathrm{e}^{\frac{1}{4 T}} \int_{0}^{t} s^{-\gamma} \mathrm{e}^{-\frac{1}{4 s}} \mathrm{~d} s\right) w^{\prime}(T) \\
& +\frac{1}{4} \int_{0}^{t} s^{-\gamma} \mathrm{e}^{-\frac{1}{4 s}} \int_{s}^{T} \sigma^{\gamma-2} \mathrm{e}^{\frac{1}{4 \sigma}} g(w(\sigma)) \mathrm{d} \sigma \mathrm{d} s .
\end{aligned}
$$


Furthermore, if $w \in C([0, T])$ is a solution of

$$
\begin{aligned}
w(t)= & w(0)+\left(T^{\gamma} \mathrm{e}^{\frac{1}{4 T}} \int_{0}^{t} s^{-\gamma} \mathrm{e}^{-\frac{1}{4 s}} \mathrm{~d} s\right) v \\
& +\frac{1}{4} \int_{0}^{t} s^{-\gamma} \mathrm{e}^{-\frac{1}{4 s}} \int_{s}^{T} \sigma^{\gamma-2} \mathrm{e}^{\frac{1}{4 \sigma}} g(w(\sigma)) \mathrm{d} \sigma \mathrm{d} s,
\end{aligned}
$$

then $w \in C^{1}((0, T])$, with $w^{\prime}(T)=v$, and is also a solution of (2.2) (and therefore in $C^{2}((0, T])$ and a solution of $\left.(2.1)\right)$.

Proof. - Without loss of generality, we may choose $T>0$ small enough so that $H^{\prime}(s) \geqslant 0$ on $(0, T]$. Indeed, this is always true if $\alpha \geqslant \frac{4}{N-2}$. Otherwise, it suffices to require that $T<\frac{1}{4(\gamma-1)}$. It follows that for all $s \in(0, T], G(w(s)) \leqslant H(s) \leqslant H(T)$. In particular, $|w(s)|$ is bounded on $(0, T]$. The integral equation (2.3), with $t_{0}=T$ for example, along with Lemma 2.1, now shows that $\lim _{t \rightarrow 0+} w(t)$ exists. Letting $t_{0} \rightarrow 0+$ in (2.3), we obtain (2.5).

Next, Eq. (2.2) and Corollary 2.2 imply that $\lim _{s \rightarrow 0+} w^{\prime}(s)$ exists and equals $g(w(0))$. Thus, $w$ extends to a $C^{1}$ function on $[0, T]$, verifying (2.4). The last statement in the proposition is obvious.

In the above proposition, the continuity of $w$ at $t=0$ corresponds to the existence of $\lim _{r \rightarrow \infty} r^{2 / \alpha} f(r)$, where $f$ and $w$ are related by (1.9), which is well known. The new aspect of the above result is the differentiability of $w$ at $t=0$ and the fact that $w^{\prime}(0)$ is determined by $w(0)$. This means in particular, that in proving local existence of solutions to the inverted profile equation (2.1), we cannot hope to specify both $w(0)$ and $w^{\prime}(0)$.

Given $\mu, v \in \mathbb{R}$ and $T>0$, we define a mapping $\mathcal{F}_{\mu, \nu}: C([0, T]) \rightarrow C([0, T])$ by

$$
\begin{aligned}
\mathcal{F}_{\mu, \nu} w(t)= & \mu+\left(T^{\gamma} \mathrm{e}^{\frac{1}{4 T}} \int_{0}^{t} s^{-\gamma} \mathrm{e}^{-\frac{1}{4 s}} \mathrm{~d} s\right) v \\
& +\frac{1}{4} \int_{0}^{t} s^{-\gamma} \mathrm{e}^{-\frac{1}{4 s}} \int_{s}^{T} \sigma^{\gamma-2} \mathrm{e}^{\frac{1}{4 \sigma}} g(w(\sigma)) \mathrm{d} \sigma \mathrm{d} s .
\end{aligned}
$$

It is clear from what has come before that $\mathcal{F}_{\mu, \nu}$ does indeed map $C([0, T])$ into itself, and that a fixed point of $\mathcal{F}_{\mu, v}$ is a solution of (2.5), with $w(0)=\mu$ and $w^{\prime}(T)=v$, and thus a solution of (2.1).

We denote by $C_{M}([0, T])$ the set of functions $w \in C([0, T])$ such that $\sup _{t \in[0, T]}|w(t)|$ $\leqslant M$.

THEOREM 2.5. - Let $R>0, M>0$ be such that $5 R<M$. There exists $T_{0}=$ $T_{0}(R, M)>0$ such that if

(i) $0<T \leqslant T_{0}$,

(ii) $|\mu| \leqslant R$,

(iii) $T^{2}|v| \leqslant R$,

then $\mathcal{F}_{\mu, \nu}$ is strict contraction on $C_{M}([0, T])$, and so has a unique fixed point in $C_{M}([0, T])$, which is a solution of $(2.5)$ with $w(0)=\mu$ and $w^{\prime}(T)=v$. 
Furthermore, there exists $C=C\left(R, M, T_{0}\right)$ such that if $\mu_{1}, v_{1}$ and $\mu_{2}, v_{2}$ are two pairs of real numbers satisfying the above conditions for the same $T \in\left(0, T_{0}\right]$, and $w_{1}$ and $w_{2}$ the two corresponding fixed points, then

$$
\begin{aligned}
& \sup _{t \in[0, T]}\left|w_{1}(t)-w_{2}(t)\right|+\sup _{t \in[0, T]}\left|w_{1}^{\prime}(t)-w_{2}^{\prime}(t)\right| \\
& \quad \leqslant C\left(\left|\mu_{1}-\mu_{2}\right|+\left|v_{1}-v_{2}\right|\right)=C\left(\left|w_{1}(0)-w_{2}(0)\right|+\left|w_{1}^{\prime}(T)-w_{2}^{\prime}(T)\right|\right) .
\end{aligned}
$$

Proof. - For $T>0$, set

$$
\begin{aligned}
& K_{1}(T)=T^{\gamma-2} \mathrm{e}^{\frac{1}{4 T}} \int_{0}^{T} s^{-\gamma} \mathrm{e}^{-\frac{1}{4 s}} \mathrm{~d} s, \\
& K_{2}(T)=\sup _{s \in(0, T]} \frac{1}{4} s^{-\gamma} \mathrm{e}^{-\frac{1}{4 s}} \int_{s}^{T} \sigma^{\gamma-2} \mathrm{e}^{\frac{1}{4 \sigma}} \mathrm{d} \sigma .
\end{aligned}
$$

By Lemmas 2.1 and 2.3 it is clear that both $K_{1}$ and $K_{2}$ can be considered as continuous functions on $[0, \infty)$, with $K_{1}(0)=4$. (Indeed, $K_{2}(T)$ is a nondecreasing function of $T>0$, and so has a limit as $T \rightarrow 0+$.) Let $\mu_{1}, v_{1}, \mu_{2}, v_{2} \in \mathbb{R}$. It follows that if $w_{1}, w_{2} \in C_{M}([0, T])$ and $t \in[0, T]$, then:

$$
\begin{aligned}
& \left|\mathcal{F}_{\mu_{1}, \nu_{1}} w_{1}(t)-\mathcal{F}_{\mu_{2}, \nu_{2}} w_{2}(t)\right| \\
& \quad \leqslant\left|\mu_{1}-\mu_{2}\right|+T^{2} K_{1}(T)\left|v_{1}-v_{2}\right|+T K_{2}(T) \sup _{t \in[0, T]}\left|g\left(w_{1}(t)\right)-g\left(w_{2}(t)\right)\right| \\
& \quad \leqslant\left|\mu_{1}-\mu_{2}\right|+T^{2} K_{1}(T)\left|v_{1}-v_{2}\right|+T K_{2}(T) D \sup _{t \in[0, T]}\left|w_{1}(t)-w_{2}(t)\right|,
\end{aligned}
$$

where $D=\sup _{|x| \leqslant M}\left|g^{\prime}(x)\right|$.

We first apply (2.8) in the case where $\mu_{2}=0, v_{2}=0, w_{2}(t) \equiv 0$, and for simplicity of notation, we suppress the subscripts in $\mu_{1}, v_{1}$ and $w_{1}$. Suppose further that conditions (ii) and (iii) in the statement hold. It follows from (2.8) that

$$
\left|\mathcal{F}_{\mu, \nu} w(t)\right| \leqslant R+K_{1}(T) R+T K_{2}(T) D M
$$

In particular, if

$$
\frac{R}{M}\left(1+K_{1}(T)\right)+T K_{2}(T) D \leqslant 1,
$$

then $\mathcal{F}_{\mu, v}$ maps $C_{M}([0, T])$ into itself. Next we apply (2.8) with $\mu_{1}=\mu_{2}=\mu$ and $v_{1}=v_{2}=v$. This gives

$$
\left|\mathcal{F}_{\mu, \nu} w_{1}(t)-\mathcal{F}_{\mu, \nu} w_{2}(t)\right| \leqslant T K_{2}(T) D \sup _{t \in[0, T]}\left|w_{1}(t)-w_{2}(t)\right|
$$

Condition (2.9) thereby further implies that $\mathcal{F}_{\mu, v}$ is a strict contraction on $C_{M}([0, T])$.

Finally, suppose (2.9) holds and that $\mu_{1}, v_{1}$ and $\mu_{2}, v_{2}$ are two pairs of real numbers satisfying conditions (ii) and (iii) in the statement. If $w_{1}$ and $w_{2}$ are the respective fixed 
points of $\mathcal{F}_{\mu_{1}, v_{1}}$ and $\mathcal{F}_{\mu_{2}, v_{2}}$, then (2.8) implies that

$$
\sup _{t \in[0, T]}\left|w_{1}(t)-w_{2}(t)\right| \leqslant \frac{\left|\mu_{1}-\mu_{2}\right|+T^{2} K_{1}(T)\left|v_{1}-v_{2}\right|}{1-T K_{2}(T) D} .
$$

To obtain a similar estimate for $\sup _{t \in[0, T]}\left|w_{1}^{\prime}(t)-w_{2}^{\prime}(t)\right|$ we use (2.2):

$$
\begin{aligned}
\sup _{s \in[0, T]}\left|w_{1}^{\prime}(s)-w_{2}^{\prime}(s)\right| \leqslant & \left(\sup _{s \in[0, T]} s^{-\gamma} \mathrm{e}^{-\frac{1}{4 s}} T^{\gamma} \mathrm{e}^{\frac{1}{4 T}}\right)\left|w_{1}^{\prime}(T)-w_{2}^{\prime}(T)\right| \\
& +K_{2}(T) D \sup _{t \in[0, T]}\left|w_{1}(t)-w_{2}(t)\right| .
\end{aligned}
$$

To conclude the proof, we just have to observe that there exists $T_{0}>0$ such that all the conditions can be verified uniformly for $T \in\left(0, T_{0}\right]$. Indeed, since $K_{1}$ and $K_{2}$ are continuous on $[0, \infty)$, and $K_{1}(0)=4$, it is clear from the assumption that $5 R<M$ that there exists $T_{0}>0$ such that (2.9) holds for all $T \in\left(0, T_{0}\right]$. Furthermore, $T^{2} K_{1}(T)$ is bounded on $\left(0, T_{0}\right]$, and since

$$
\lim _{T \rightarrow 0+} \sup _{s \in[0, T]} s^{-\gamma} \mathrm{e}^{-\frac{1}{4 s}} T^{\gamma} \mathrm{e}^{\frac{1}{4 T}}=1
$$

so is $\sup _{s \in[0, T]} S^{-\gamma} \mathrm{e}^{-\frac{1}{4 s}} T^{\gamma} \mathrm{e}^{\frac{1}{4 T}}$.

Remark 2.6. - It is clear from the previous proof that if $5 R<M_{1}<M_{2}$, then there exists $T_{0}=T_{0}\left(R, M_{1}, M_{2}\right)$ such that the conclusions of Theorem 2.5 are valid for all $M \in\left[M_{1}, M_{2}\right]$ with the same value of $T_{0}$. Indeed, it suffices that (2.9) hold for all $T \in\left(0, T_{0}\right]$ and all $M \in\left[M_{1}, M_{2}\right]$, which is clearly possible. The advantage of so choosing $T_{0}$ is that Theorem 2.5 thereby gives existence of solutions to (2.5) in $C_{M_{1}}([0, T])$ and uniqueness of solutions in the larger space $C_{M_{2}}([0, T])$. We will use this fact in the proof of the following proposition, which plays a fundamental role in the proofs of Theorems 1.3 and 1.4 in Section 4 below.

Proposition 2.7. - Let $R>0, M>0$ (with $5 R<M$ ) and $T_{0}=T_{0}(R, M)>0$ be as in the statement of Theorem 2.5, and suppose (as per the previous remark) that the same value of $T_{0}$ is valid in Theorem 2.5 with $M$ replaced by $M+1$. Let $\mu, v$ and $T \in\left(0, T_{0}\right]$ satisfy conditions (i), (ii), and (iii) in the statement of Theorem 2.5. Let $w \in C_{M}([0, T]) \cap C^{2}((0, T]) \cap C^{1}([0, T])$ be the resulting solution of $(2.5)$ with $w(0)=\mu$ and $w^{\prime}(T)=v$, and suppose that $w$ is not an identically constant solution. It follows that:

(i) there is at most one value of $s \in[0, T]$ such that $w^{\prime}(s)=0$;

(ii) if $N>2, \alpha>\frac{2}{N-2}$ and $w(0)>0$, there is at most one value of $s \in[0, T]$ such that $w(s)=\beta^{1 / \alpha}$.

Proof. - (i) We consider first the case where $g(\mu)=0$. By Proposition 2.4, we know that $w^{\prime}(0)=0$. Suppose that there exists $T_{1} \in(0, T]$ such that $w^{\prime}\left(T_{1}\right)=0$. We apply the uniqueness part of Theorem 2.5 on the interval $\left[0, T_{1}\right] \subset[0, T]$. Since $g(\mu)=0$, one solution of (2.5) with $w(0)=\mu$ and $w^{\prime}\left(T_{1}\right)=0$ is the constant solution equal to $\mu$. It follows that $w$ must be that solution on $\left[0, T_{1}\right]$, and therefore by local uniqueness 
of solutions to (1.12) starting from any positive point, $w$ is identically constant for all $s \in[0, T]$.

We consider next the case where $g(\mu) \neq 0$, and so $w^{\prime}(0) \neq 0$. Suppose there exist $0<T_{1}<T_{2} \leqslant T$ such that $w^{\prime}\left(T_{1}\right)=w^{\prime}\left(T_{2}\right)=0$, and that $w^{\prime}(s) \neq 0, \forall s \in\left(T_{1}, T_{2}\right)$. For every $\tau \in(0, T]$, let $w_{\tau}$ be the solution constructed by the fixed point argument in Theorem 2.5 with $\left|w_{\tau}(s)\right| \leqslant M$ on $[0, \tau], w_{\tau}(0)=\mu$ and $w_{\tau}^{\prime}(\tau)=0$. By uniqueness, it follows that $w_{T_{1}}=w$ on $\left[0, T_{1}\right]$ and $w_{T_{2}}=w$ on $\left[0, T_{2}\right]$. It is clear that $w_{\tau}(\tau)$ can never be equal to a zero of $g$. Indeed, that would mean $g\left(w_{\tau}(\tau)\right)=0$ and $w_{\tau}^{\prime}(\tau)=0$, and so by local uniqueness (starting at $s=\tau$ ) it would follow that $w_{\tau}(s) \equiv w_{\tau}(\tau), \forall s \in[0, \tau]$. Since (as we show below) $w_{\tau}(\tau)$ is a continuous function of $\tau \in(0, T]$, it follows that $g\left(w_{\tau}(\tau)\right)$ must all be of the same sign, and in particular $g\left(w\left(T_{1}\right)\right)$ and $g\left(w\left(T_{2}\right)\right)$ have the same sign. By (1.12), this implies that $w^{\prime \prime}\left(T_{1}\right)$ and $w^{\prime \prime}\left(T_{2}\right)$ have the same sign; but this is impossible since $T_{1}$ and $T_{2}$ are successive zeroes of $w^{\prime}$.

To prove that $w_{\tau}(\tau)$ is a continuous function of $\tau \in(0, T]$, we fix $\tau_{0} \in(0, T]$ and suppose that $\tau \rightarrow \tau_{0}$ in $(0, T]$. We claim that if $\tau$ is close enough to $\tau_{0}$, then $w_{\tau_{0}}$ can be defined on $[0, \tau]$ and obtained from Theorem 2.5 using the data $\mu=w_{\tau_{0}}(0)$ and $v=w_{\tau_{0}}^{\prime}(\tau)$. This is clear in the case $\tau<\tau_{0}$. If $\tau>\tau_{0}$ (and so necessarily $\tau_{0}<T$ ), we note that by local existence and uniqueness starting at $\tau_{0}, w_{\tau_{0}}$ can be continued as a solution on $\left[0, \tau_{0}+\varepsilon\right]$ for some $\varepsilon>0$. Moreover, if $\varepsilon$ is small enough, it follows that $s^{2}\left|w_{\tau_{0}}^{\prime}(s)\right| \leqslant R, \forall s \in\left[\tau_{0}-\varepsilon, \tau_{0}+\varepsilon\right]$ and $\left|w_{\tau_{0}}(s)\right| \leqslant M+1, \forall s \in\left[0, \tau_{0}+\varepsilon\right]$. By uniqueness of solutions in $C_{M+1}([0, \tau])$, it follows that $w_{\tau_{0}}$ is indeed the solution obtained from Theorem 2.5 on $[0, \tau]$ with the data $\mu=w_{\tau_{0}}(0)$ and $v=w_{\tau_{0}}^{\prime}(\tau)$. Next, we write

$$
w_{\tau}(\tau)-w_{\tau_{0}}\left(\tau_{0}\right)=w_{\tau}(\tau)-w_{\tau_{0}}(\tau)+w_{\tau_{0}}(\tau)-w_{\tau_{0}}\left(\tau_{0}\right) .
$$

On the one hand, since $w_{\tau_{0}}$ is continuous, we know that $w_{\tau_{0}}(\tau) \rightarrow w_{\tau_{0}}\left(\tau_{0}\right)$ as $\tau \rightarrow \tau_{0}$. On the other hand, (2.7) applied to $w_{\tau}$ and $w_{\tau_{0}}$ on the interval $[0, \tau]$ implies that

$$
\left|w_{\tau}(\tau)-w_{\tau_{0}}(\tau)\right| \leqslant C\left|w_{\tau_{0}}^{\prime}(\tau)\right|
$$

where $C$ depends only on $T_{0}$, not on $\tau$. Since $w_{\tau_{0}}^{\prime}(\tau) \rightarrow 0$ as $\tau \rightarrow \tau_{0}$, we conclude that $w_{\tau}(\tau) \rightarrow w_{\tau_{0}}\left(\tau_{0}\right)$.

(ii) Suppose first that $w(0)=\beta^{1 / \alpha}$. The first part of the proposition implies that $w$ is either decreasing or increasing on $(0, T]$, and thus cannot equal $\beta^{1 / \alpha}$ at any $s \in(0, T]$.

Suppose next that $0<w(0)<\beta^{1 / \alpha}$, and that there exist $0<s_{1}<s_{2} \leqslant T$ such that $w\left(s_{1}\right)=w\left(s_{2}\right)=\beta^{1 / \alpha}$. Since $w^{\prime}(0)<0$ (by Proposition 2.4), it follows that $w^{\prime}$ must have a zero on each of the intervals $\left(0, s_{1}\right)$ and $\left(s_{1}, s_{2}\right)$, contradicting the first part of the proposition. The case $w(0)>\beta^{1 / \alpha}$ is handled similarly.

Remark 2.8. - As used implicitly in the previous proof, a given solution of (2.5) can be obtained from Theorem 2.5 on intervals $[0, T]$, with different values of $T$. Obviously, the data $v=w^{\prime}(T)$ will change as $T$ changes, in order to produce the same solution. Furthermore, it is clear from Proposition 2.4 that any solution of (1.12) on an interval $\left(0, T^{\prime}\right)$ can be obtained via Theorem 2.5 . In other words, given any solution on $\left(0, T^{\prime}\right)$, the part of that solution on $(0, T), T \leqslant T_{0}$, can be obtained as a result of the contraction mapping argument used in the proof of Theorem 2.5 for sufficiently small $T_{0}$. Indeed, 
given finitely many such solutions, one can choose the parameters $R, M$ and $T_{0}$ in Theorem 2.5 so that all of these solutions will be produced in the same manner via Theorem 2.5, for all $T \in\left(0, T_{0}\right]$ with appropriate choices of $\mu$ and $\nu$.

This is also true for any collection $\mathcal{E}$ of solutions of (1.12) defined on a common interval $\left(0, T_{1}\right)$ for which $H_{w, w^{\prime}}(T)$ ranges over a bounded set for $w \in \mathcal{E}$, for some fixed $T \in\left(0, T_{1}\right)$. To see this note first that by continuous dependence starting at any $s>0$, it follows that $H\left(T^{\prime}\right)$ ranges over a bounded set for any fixed $T^{\prime} \in\left(0, T_{1}\right)$. Thus, by choosing $T^{\prime} \in\left(0, T_{1}\right)$ small enough (in the case $\left.\gamma>1\right)$, we may assume that $H$ is nondecreasing on $\left[0, T^{\prime}\right]$. Suppose that $H_{w, w^{\prime}}\left(T^{\prime}\right) \leqslant D$ for all $w \in \mathcal{E}$. It follows that $G(w(s)) \leqslant D$ and $2 s^{4} w^{\prime}(s)^{2} \leqslant s^{2}\left(D-G_{\min }\right)$, for all $w \in \mathcal{E}$ and all $s \in\left[0, T^{\prime}\right]$. It is therefore clear that there exist $R, M$ and $T_{0}$ (with $5 R<M$ ) such that for all $w \in \mathcal{E}$,

$$
\begin{aligned}
& |w(0)| \leqslant R, \\
& T^{2}\left|w^{\prime}(T)\right| \leqslant R, \quad \forall T \in\left[0, T_{0}\right], \\
& |w(s)| \leqslant M, \quad \forall s \in\left[0, T_{0}\right] .
\end{aligned}
$$

By choosing $T_{0}>0$ smaller if necessary, it is clear that all the solutions $w \in \mathcal{E}$ can be obtained from Theorem 2.5 for the same values of $R, M$ and $T_{0}$, and on all subintervals $[0, T] \subset\left[0, T_{0}\right]$.

It is also worth noting that, with the exception of the second part of Proposition 2.7, the results of this section are all valid if $\gamma$ is considered as an arbitrary but fixed real number and if $g$ is replaced by an arbitrary locally Lipschitz function $g: \mathbb{R} \rightarrow \mathbb{R}$, with primitive $G$ such that $G(0)=0$ and $\lim _{|x| \rightarrow \infty} G(x)=\infty$.

\section{Global existence and asymptotic behvavior of the inverted profile}

In this section we show that the solutions $w(s)$ of (2.5), and therefore (1.12), shown to exist in Theorem 2.5 can be continued for all $s>0$ and study their behvavior as $s \rightarrow \infty$.

Proposition 3.1. - Let $w:(0, T) \rightarrow \mathbb{R}$ be a solution of (1.12). Then $w$ can be (uniquely) continued as a solution of $(1.12)$ on $(0, \infty)$.

(i) If $\alpha \leqslant \frac{4}{N-2}$, then $|w(s)|$ and $\left|s w^{\prime}(s)\right|$ are bounded on $(0, \infty)$.

(ii) If $\alpha>\frac{4}{N-2}$, then $H(s) \leqslant C\left(1+s^{2(1-\gamma)}\right)=C\left(1+s^{N-2-4 / \alpha}\right)$, where $H$ is the functional defined by (1.13). In particular, $\left|s^{\gamma} w^{\prime}(s)\right|$ is bounded as $s \rightarrow \infty$.

Proof. - We need to obtain an a priori bound on $w(s)$ and $w^{\prime}(s)$ on any finite interval. We note that by (1.13) and (1.14),

$$
H^{\prime}(s)=w^{\prime}(s)^{2}[1-4(\gamma-1) s]=[H(s)-G(s)] \frac{1-4(\gamma-1) s}{2 s^{2}} .
$$

We consider first the case $\alpha \leqslant \frac{4}{N-2}$, and so $\gamma \geqslant 1$.

$$
H^{\prime}(s) \leqslant[H(s)-G(s)] \frac{1}{2 s^{2}} \leqslant\left[H(s)-G_{\min }+1\right] \frac{1}{2 s^{2}},
$$


and so

$$
\frac{H^{\prime}(s)}{H(s)-G_{\min }+1} \leqslant \frac{1}{2 s^{2}} .
$$

Integrating this last relationship gives an a priori bound on $H(s)$ on any interval $(\varepsilon, \infty)$. This completes the proof in the case $\alpha \leqslant \frac{4}{N-2}$. (We have already observed that $H^{\prime}(s) \geqslant 0$ for small $s>0$, and so $H(s)$ is bounded on $(0, \varepsilon)$.)

If $\alpha>\frac{4}{N-2}$, the above calculation is modified to give:

$$
\frac{H^{\prime}(s)}{H(s)-G_{\min }+1} \leqslant \frac{1+4(1-\gamma) s}{2 s^{2}} .
$$

When integrated, this shows that $H(s)$ stays bounded on any bounded interval, proving that $w(s)$ can be continued for all $s>0$, and gives the growth estimate claimed in the statement.

Proposition 3.2. - If $w:(0, \infty) \rightarrow \mathbb{R}$ is a solution of $(1.12)$ such that $\lim _{s \rightarrow \infty} w(s)$ $=l \in \mathbb{R}$ exists, then $g(l)=0$.

Proof. - The proof is based on Eq. (2.1), and we treat separately the three cases $\alpha<\frac{4}{N-2}, \alpha=\frac{4}{N-2}$, and $\alpha>\frac{4}{N-2}$.

In the first case, where $\gamma>1$, if $g(l) \neq 0$, then the right-hand side of (2.1) is not integrable as $s \rightarrow \infty$. We deduce that $s^{\gamma} \mathrm{e}^{\frac{1}{4 s}} w^{\prime}(s)$ behaves like $C s^{\gamma-1}$ as $s \rightarrow \infty$ for some $C \neq 0$, and so $w^{\prime}(s)$ behaves like $C s^{-1}$. This contradicts the hypothesis that $\lim _{s \rightarrow \infty} w(s)=l \in \mathbb{R}$ exists.

In the second case, where $\gamma=1$, if $g(l) \neq 0$, then the right-hand side of (2.1) is still not integrable as $s \rightarrow \infty$. We deduce that $s \mathrm{e}^{\frac{1}{4 s}} w^{\prime}(s)$ behaves like $C \log s$ as $s \rightarrow \infty$, and so $w^{\prime}(s)$ behaves like $C \frac{\log s}{s}$, which is likewise not integrable as $s \rightarrow \infty$. This again contradicts the hypothesis that $\lim _{s \rightarrow \infty} w(s)=l \in \mathbb{R}$ exists.

In the third case, since $\gamma<1$, we see that the right-hand side of (2.1) is integrable as $s \rightarrow \infty$. Thus, $\lim _{s \rightarrow \infty} s^{\gamma} \mathrm{e}^{\frac{1}{4 s}} w^{\prime}(s)=m \in \mathbb{R}$ exists. This limit must in fact be 0 , since otherwise $w^{\prime}(s)$ would decay as $m s^{-\gamma}$, and itself would not be integrable as $s \rightarrow \infty$, contradicting the hypothesis that $\lim _{s \rightarrow \infty} w(s)=l \in \mathbb{R}$ exists. Integrating (2.1) from $s$ to $\infty$, taking into account $m=0$, we obtain

$$
4 s^{\gamma} \mathrm{e}^{\frac{1}{4 s}} w^{\prime}(s)=\int_{s}^{\infty} t^{\gamma-2} \mathrm{e}^{\frac{1}{4 t}} g(w(t)) \mathrm{d} t .
$$

If $g(l) \neq 0$, then the above integral behaves like $C s^{\gamma-1}$ as $s \rightarrow \infty$, and so $w^{\prime}(s)$ behaves like $C s^{-1}$. This last contradiction proves that $g(l)=0$.

PROPOSITION 3.3. - Let $w:(0, \infty) \rightarrow \mathbb{R}$ be a solution of $(1.12)$, where $\alpha \neq \frac{4}{N-2}$. If $\alpha>\frac{4}{N-2}$, suppose in addition that $w$ is bounded on $(0, \infty)$. (This is true automatically if $\alpha<\frac{4}{N-2}$ by Proposition 3.1.) It follows that $\lim _{s \rightarrow \infty} w(s)=l \in \mathbb{R}$ exists and that $\lim _{s \rightarrow \infty} H(s)=G(l)$.

In the case $\alpha>\frac{4}{N-2}$, it must be that $G(l) \geqslant G(w(0))$; and if $G(l)=G_{\min }$, then $w(s) \equiv l$. 
Proof. - We will prove this result by proving in fact that $\lim _{s \rightarrow \infty} G(w(s))$ exists (and is finite). It follows from (1.14) that $H(s)$ is a monotone function for large $s$, nonincreasing if $\alpha<\frac{4}{N-2}$, nondecreasing if $\alpha \geqslant \frac{4}{N-2}$. Thus, the following limit exists:

$$
\lim _{s \rightarrow \infty} H(s)=H_{\infty}
$$

In the former case, since $H$ is bounded below, we conclude immediately that $H_{\infty} \in \mathbb{R}$. The same is true in the latter case. Indeed, if $H_{\infty}=\infty$, since $w$ is bounded on $(0, \infty)$, it would follow that $\lim _{s \rightarrow \infty} s^{2} w^{\prime}(s)^{2}=\infty$, contradicting the boundedness of $w$ on $(0, \infty)$. Thus $H_{\infty}$ is finite. As a consequence, (1.13) implies that $s^{2} w^{\prime}(s)^{2}$ is bounded on $(0, \infty)$, and (1.14) implies that $s w^{\prime}(s)^{2}$ is integrable as $s \rightarrow \infty\left(\right.$ since $\left.\alpha \neq \frac{4}{N-2}\right)$.

It is also clear that

$$
\liminf _{s \rightarrow \infty} s^{2} w^{\prime}(s)^{2}=0
$$

since otherwise $w$ could not be bounded. It follows that

$$
\limsup _{s \rightarrow \infty} G(w(s))=H_{\infty}
$$

We now wish to show that $\liminf _{s \rightarrow \infty} G(w(s))=H_{\infty}$. Suppose not. To fix the notation, let $M=\sup _{s \geqslant 0}|w(s)|, D=\max _{|x| \leqslant M}|g(x)|$, and $K=\sup _{s \geqslant 0} s\left|w^{\prime}(s)\right|$. Since $\liminf _{s \rightarrow \infty} G(w(s))<H_{\infty}$, there exists $\varepsilon>0$ and a sequence $\left\{s_{k}\right\}_{k=1,2,3, \ldots}$ such that

(i) $s_{k+1} \geqslant s_{k} \mathrm{e}^{\varepsilon / 4 D K}$,

(ii) $G\left(w\left(s_{k}\right)\right) \leqslant H_{\infty}-\varepsilon$,

(iii) $H(s) \geqslant H_{\infty}-\frac{\varepsilon}{4}$ for all $s \geqslant s_{1}$.

If $s>s_{k}$, it follows that

$$
\left|G(w(s))-G\left(w\left(s_{k}\right)\right)\right| \leqslant \int_{s_{k}}^{s}|g(w(\sigma))|\left|w^{\prime}(\sigma)\right| \mathrm{d} \sigma \leqslant D K \int_{s_{k}}^{s} \frac{1}{\sigma} \mathrm{d} \sigma=D K \log \frac{s}{s_{k}} .
$$

Thus, if $s \in\left[s_{k}, s_{k} \mathrm{e}^{\varepsilon / 4 D K}\right]$, then

$$
G(w(s)) \leqslant G\left(w\left(s_{k}\right)\right)+\frac{\varepsilon}{4} \leqslant H_{\infty}-\frac{3 \varepsilon}{4}
$$

and so

$$
2 s^{2} w^{\prime}(s)^{2}=H(s)-G(w(s)) \geqslant H_{\infty}-\frac{\varepsilon}{4}-H_{\infty}+\frac{3 \varepsilon}{4}=\frac{\varepsilon}{2} .
$$

It follows that

$$
\int_{s_{k}}^{s_{k+1}} s w^{\prime}(s)^{2} \mathrm{~d} s \geqslant \int_{s_{k}}^{s_{k} \mathrm{e}^{\varepsilon / 4 D K}} s w^{\prime}(s)^{2} \mathrm{~d} s \geqslant \frac{\varepsilon}{4} \int_{s_{k}}^{s_{k} \mathrm{e}^{\varepsilon / 4 D K}} \frac{1}{s} \mathrm{~d} s=\frac{\varepsilon^{2}}{16 D K},
$$

which contradicts the fact that $s w^{\prime}(s)^{2}$ is integrable as $s \rightarrow \infty$. 
We have therefore proved that

$$
\lim _{s \rightarrow \infty} G(w(s))=H_{\infty}
$$

which implies that $\lim _{s \rightarrow \infty} w(s)=l$ exists (and is finite). Moreover, we note that

$$
H_{\infty}=G(l)
$$

and

$$
\lim _{s \rightarrow \infty} s^{2} w^{\prime}(s)^{2}=0 .
$$

To prove the last statement in the proposition, we recall that $H^{\prime}(s) \geqslant 0$ for all $s>0$ when $\alpha \geqslant \frac{4}{N-2}$. Thus $G(w(0))=H(0) \leqslant H(s) \leqslant H_{\infty}=G(l)$. Moreover, if $G(l)=G_{\min }$, then $G(w(s)) \geqslant G_{\min }=G(l)$. Thus, $2 s^{2} w^{\prime}(s)^{2}=H(s)-G(w(s)) \leqslant$ $G(l)-G(l)=0$.

It is worth noting that the conclusion of the previous proposition is false if $\alpha=\frac{4}{N-2}$. Indeed, $H$ is a nondecreasing function, which therefore has a $\operatorname{limit}_{\lim } \rightarrow \infty H(s)=H_{\infty}$. If $\lim _{s \rightarrow \infty} w(s)=l \in \mathbb{R}$, then $s w^{\prime}(s)$ must also have a limit, which must be zero since $w$ is bounded. It follows that $H_{\infty}=G(l) \in \mathbb{R}$. Furthermore, since $H$ is nondecreasing, it is clear that $G(w(0))=H(0) \leqslant H_{\infty}=G(l)$. By Proposition 3.2, it must also be that $g(l)=0$. This is impossible if $G(w(0))$ is bigger than all the values of $G$ on the zeroes of $g$. Since all solutions are bounded in this case, it follows that there are indeed many bounded solutions which do not converge as $s \rightarrow \infty$.

The following proposition is not directly needed for the proof of Theorem 1.4 in the next section. It is, however, of some independent interest, and played and important role in our investigations.

PROPOSITION 3.4. - If $\alpha>\frac{4}{N-2}$, i.e. $\gamma<1$, there is no solution $w$ of (1.12) such that $w(s)>0$ and $g(w(s))>0$ for all sufficiently large $s>0$. In particular, any unbounded solution must have infinitely many zeroes.

Proof. - We prove the first statement by contradiction. Thus, we suppose that $w$ is indeed a solution such that $w(s)>0$ and $g(w(s))>0$ for all sufficiently large $s>0$. We claim that $w^{\prime}(s)>0$ for sufficiently large $s$. Indeed, at any point $s$ where $w^{\prime}(s)=0$, we have $w^{\prime \prime}(s)=-g(w(s))$, which is negative for large $s>0$. Since $w$ cannot have two successive strict local maximums, $w^{\prime}(s)$ must either ultimately be positive or negative. It $w^{\prime}(s)<0$ for large $s>0$, then $w$ must be bounded (since it is positive) and have a finite limit. By Proposition 3.3, since $w$ is not a constant solution, this limit must be 0 , contradicting the fact that $g(w(s))>0$ for large $s$. This proves the claim.

Since $w^{\prime}(s)>0$ for large $s>0, g(w(s))$ is increasing for large $s$. Integrating formula (2.1) from $s$ to $T$ and letting $T \rightarrow \infty$, we obtain (for large $s>0$ )

$$
4 s^{\gamma} \mathrm{e}^{\frac{1}{4 s}} w^{\prime}(s) \geqslant \int_{s}^{\infty} t^{\gamma-2} \mathrm{e}^{\frac{1}{4 t}} g(w(t)) \mathrm{d} t \geqslant g(w(s)) \int_{s}^{\infty} t^{\gamma-2} \mathrm{~d} t=g(w(s)) \frac{s^{\gamma-1}}{1-\gamma} .
$$


In other words, for large $s>0$,

$$
\frac{w^{\prime}(s)}{g(w(s))} \geqslant \frac{C}{s}
$$

which implies that $w(s)$ blows up in finite time, contradicting Proposition 3.1.

To prove the second part of the proposition, consider an unbounded solution with only finitely many zeroes. Without loss of generality, we may suppose that $w(s)>0$ for sufficiently large $s$. Since $w$ is unbounded, it follows that $H(s)$, which is nondecreasing, tends to $\infty$ as $s \rightarrow \infty$, and so $H(s)>0$ for sufficiently large $s$. In particular, for $s$ sufficiently large $w^{\prime}(s)$ cannot have a zero in the range where $G(w(s)) \leqslant 0$. Since $w(s)>0$, this implies that $w^{\prime}(s)$ can equal zero only if $g(w(s))>0$, and so $w^{\prime \prime}(s)$ would be negative at such a point. Since $w$ is unbounded, this is impossible, and so $w^{\prime}(s)>0$ for sufficiently large $s$. It follows that $g(w(s))>0$ for large $s$, contradicting the first part of the proposition.

This proves the proposition.

PROPOSITION 3.5. - Suppose $\alpha>\frac{2}{N-2}$ and let $w$ be a solution of $(1.12)$ on $(0, \infty)$ such that $w(s)>0$ for all $s>0$ and $w(s) \rightarrow 0$ as $s \rightarrow \infty$. Then $\lim _{s \rightarrow \infty} s^{1 / \alpha} w(s)$ exists and is finite. In particular, if $f$ is related to $w$ by (1.9) and if $u$ is the resulting self-similar solution of (1.1) given by (1.5), then $u$ is regular for $t>0$ and $x \in \mathbb{R}^{N}$.

Proof. - We set

$$
h(s)=\frac{s w^{\prime}(s)}{w(s)}+\frac{1}{\alpha} .
$$

Since $w^{\prime}(s)$ cannot have successive zeroes without $g(w(s))$ changing sign, it follows that $w^{\prime}(s)<0$ for large $s$. In particular, $h(s)<\frac{1}{\alpha}$ for large $s$. A tedious calculation shows that

$$
\frac{\mathrm{d}}{\mathrm{d} s}\left(s^{-\frac{N-2}{2}} \mathrm{e}^{\frac{1}{4 s}} h(s)\right)=-s^{-\frac{N}{2}} \mathrm{e}^{\frac{1}{4 s}}\left(\frac{1}{4 \alpha s}+\frac{|w(s)|^{\alpha}}{4}+h(s)^{2}\right)
$$

Thus $s^{-\frac{N-2}{2}} \mathrm{e}^{\frac{1}{4 s}} h(s)$ is decreasing and

$$
\lim _{s \rightarrow \infty} s^{-\frac{N-2}{2}} \mathrm{e}^{\frac{1}{4 s}} h(s)=m<\infty
$$

exists. We claim that in fact $m=0$.

We can easily eliminate the case $m>0$, since then $h(s) \rightarrow \infty$ as $s \rightarrow \infty$, contradicting the fact $h(s)<1 / \alpha$ for large $s$. If $m<0$, then clearly $h(s)$, and likewise $s w^{\prime}(s) / w(s)$, converge to $-\infty$ as $s \rightarrow \infty$. Moreover, since both $w(s)$ and $H(s)$ converge as $s \rightarrow \infty$, it follows that $s^{2} w^{\prime}(s)^{2}$ must likewise converge; and its limit must be zero, since otherwise $w$ would not be bounded. Thus, $s w^{\prime}(s) / w(s)$ is indeterminant of the form $\frac{0}{0}$ as $s \rightarrow \infty$. If we then apply l'Hôpital's rule, we get that $s w^{\prime}(s) / w(s)$ converges to a finite number. This contradiction eliminates the possibility that $m<0$.

Thus

$$
\lim _{s \rightarrow \infty} s^{-\frac{N-2}{2}} \mathrm{e}^{\frac{1}{4 s}} h(s)=0
$$


and $h(s)>0, \forall s>0$. Integrating (3.1), taking (3.2) into account, we obtain

$$
\begin{aligned}
t^{-\frac{N-2}{2}} \mathrm{e}^{\frac{1}{4 t}} h(t) & =\int_{t}^{\infty} s^{-\frac{N}{2}} \mathrm{e}^{\frac{1}{4 s}}\left(\frac{1}{4 \alpha s}+\frac{|w(s)|^{\alpha}}{4}+h(s)^{2}\right) \mathrm{d} s \\
& \leqslant \mathrm{e}^{\frac{1}{4 t}}\left(\frac{1}{4 \alpha t}+\frac{|w(t)|^{\alpha}}{4}+\sup _{s \geqslant t} h(s)^{2}\right) \int_{t}^{\infty} s^{-\frac{N}{2}} \mathrm{~d} s \\
& =\frac{2}{N-2} t^{-\frac{N-2}{2}} \mathrm{e}^{\frac{1}{4 t}}\left(\frac{1}{4 \alpha t}+\frac{|w(t)|^{\alpha}}{4}+\sup _{s \geqslant t} h(s)^{2}\right) .
\end{aligned}
$$

Since $h(s)<1 / \alpha$ for large $s>0$, we see that for large $t>0$,

$$
h(t) \leqslant \frac{1}{2(N-2) \alpha t}+\frac{|w(t)|^{\alpha}}{2(N-2)}+\frac{2}{(N-2) \alpha} \sup _{s \geqslant t} h(s),
$$

and so

$$
\sup _{s \geqslant t} h(s) \leqslant \frac{\frac{1}{t}+\alpha|w(t)|^{\alpha}}{2(N-2) \alpha-4} .
$$

We immediately conclude that $h(s) \rightarrow 0$ as $s \rightarrow \infty$. From this fact it follows that $s w^{\prime}(s)+\left(\frac{1}{\alpha}-\varepsilon\right) w(s) \leqslant 0$ for large $s>0$, i.e. $\left(s^{(1 / \alpha-\varepsilon)} w(s)\right)^{\prime} \leqslant 0$, from which we see that $w(s)=\mathrm{O}\left(s^{-(1 / \alpha-\varepsilon)}\right)$ as $s \rightarrow \infty$. Putting this estimate back into (3.3), we get that $h(s)=\mathrm{O}\left(s^{-(1-\varepsilon)}\right)$ as $s \rightarrow \infty$, i.e.

$$
\frac{w^{\prime}(s)}{w(s)}=-\frac{1}{\alpha s}+\mathrm{O}\left(s^{-(2-\varepsilon)}\right) .
$$

This implies that $\lim _{s \rightarrow \infty} s^{1 / \alpha} w(s)$ exists and is finite. It follows that $f(r)$ has a finite limit as $r \rightarrow 0$, and the regularity of $f$ at $r=0$ follows from the integral equation corresponding to (1.6), as in Section 3 of [4].

\section{Existence of the regular self-similar solutions}

Proposition 4.1. - Suppose $N>2$ and $\alpha>\frac{2}{N-2}$, and let $\mu \geqslant \beta^{1 / \alpha}$. There exists a solution of (2.5) with $w(0)=\mu$, such that for some $s_{0}$ and $s_{1}$ with $0 \leqslant s_{0}<s_{1}$,

(i) $w^{\prime}(s)>0, \forall s \in\left(0, s_{0}\right)$;

(ii) $w^{\prime}(s)<0, \forall s \in\left(s_{0}, s_{1}\right]$;

(iii) $w\left(s_{1}\right)=0$;

(iv) $s_{0}=0 \Leftrightarrow \mu=\beta^{1 / \alpha}$;

(v) $w$ has precisely one intersection on $\left[0, s_{1}\right]$ with the constant solution $\beta^{1 / \alpha}$.

Proof. - Let $R>0, M>0$, and $T_{0}$ be as in the statement of Theorem 2.5, with $R>\mu$. In addition, if $\alpha<\frac{4}{N-2}$ we require $T_{0}<\frac{1}{4(\gamma-1)}$, so that by $(1.14), H^{\prime}(s) \geqslant 0$ for any solution of (2.5) on $[0, T] \subset\left[0, T_{0}\right]$.

We apply Theorem 2.5 with $\mu \geqslant \beta^{1 / \alpha}$, and we impose the following additional restrictions on $T \in\left(0, T_{0}\right)$ and $v$ : 


$$
\begin{aligned}
& \frac{R^{2}}{2 T^{2}}+G_{\min }-\max [0, G(M)]>\frac{2 T_{0}^{2} M^{2}}{\left(T_{0}-T\right)^{2}}, \\
& v \leqslant-\frac{R}{2 T^{2}} .
\end{aligned}
$$

Let $w$ be the resulting solution of (2.5), i.e. with $w(0)=\mu, w^{\prime}(T)=v$, and $|w(s)| \leqslant M$ on $[0, T]$. By Proposition 2.4, we also know that $w^{\prime}(0) \geqslant 0$, with equality if and only if $\mu=\beta^{1 / \alpha}$. If in fact $\mu=\beta^{1 / \alpha}$, it follows from Propsition 2.7 that $w^{\prime}(s)<0, \forall s \in(0, T]$. On the other hand, if $\mu>\beta^{1 / \alpha}$, then since $w^{\prime}(0)>0$ and $w^{\prime}(T)<0$, it follows from Proposition 2.7 that there exists $s_{0} \in(0, T)$ such that $w^{\prime}(s)>0, \forall s \in\left(0, s_{0}\right)$ and $w^{\prime}(s)<0, \forall s \in\left(s_{0}, T\right]$. In either case, if $w(T) \leqslant 0$, we are done. Thus, we suppose that $w(T)>0$.

It is clear that if for some $T_{1} \in\left(T, T_{0}\right], w(s) \geqslant 0$ on $\left(s_{0}, T_{1}\right]$, then $w^{\prime}(s)<0$ on $\left(s_{0}, T_{1}\right]$. Indeed, suppose $\tau \in\left(T, T_{1}\right]$ is the smallest value where $w^{\prime}(\tau)=0$. Then $0 \leqslant w(s) \leqslant w\left(s_{0}\right) \leqslant M$ on $(0, \tau]$. By Proposition 2.7 we cannot have both $w^{\prime}\left(s_{0}\right)=0$ and $w^{\prime}(\tau)=0$. It also follows that $G(w(s)) \leqslant \max [0, G(M)]$ on $\left(s_{0}, T_{1}\right]$. Since

$$
H(T)=2 T^{2} w^{\prime}(T)^{2}+G(w(T)) \geqslant \frac{R^{2}}{2 T^{2}}+G(w(T)) \geqslant \frac{R^{2}}{2 T^{2}}+G_{\min },
$$

it follows that,

$$
H(s) \geqslant \frac{R^{2}}{2 T^{2}}+G_{\min }, \quad \forall s \in\left[T, T_{0}\right] .
$$

Suppose now that $w(s)>0$ on $\left[0, T_{0}\right]$. It follows that for $s \in\left[T, T_{0}\right]$,

$w^{\prime}(s)^{2}=\frac{1}{2 s^{2}}(H(s)-G(w(s))) \geqslant \frac{1}{2 T_{0}^{2}}\left(\frac{R^{2}}{2 T^{2}}+G_{\min }-\max [0, G(M)]\right)>\frac{M^{2}}{\left(T_{0}-T\right)^{2}}$.

In other words,

$$
w^{\prime}(s) \leqslant \frac{-M}{T_{0}-T}
$$

Finally, we see that

$$
w\left(T_{0}\right) \leqslant w(T)+\left(T_{0}-T\right) \max _{s \in\left[T, T_{0}\right]} w^{\prime}(s)<M-M=0 .
$$

This concludes the proof.

LEMmA 4.2. - Suppose $\frac{2}{N-2}<\alpha<\frac{4}{N-2}$, and let $w$ be a solution of (1.12) such that $w(s)>0$ for large $s>0$. It follows that $\lim _{s \rightarrow \infty} w(s)=\beta^{1 / \alpha}$ if and only if $H(s)<0$ for sufficiently large $s>0$.

Proof. - It follows from Propositions 3.2 and 3.3 that either $\lim _{s \rightarrow \infty} w(s)=\beta^{1 / \alpha}$, in which case

$$
\lim _{s \rightarrow \infty} H(s)=G\left(\beta^{1 / \alpha}\right)=G_{\min }<0,
$$

or $\lim _{s \rightarrow \infty} w(s)=0$, in which case $\lim _{s \rightarrow \infty} H(s)=0$. The result follows since $H(s)$ is nonincreasing for large $s>0$. 
COROLlARY 4.3. - Assume $\frac{2}{N-2}<\alpha<\frac{4}{N-2}$. There exists $\varepsilon>0$ such that for every $\mu, \beta^{1 / \alpha}<\mu<(1+\varepsilon) \beta^{1 / \alpha}$, there is a solution $w$ of $(1.12)$ with $w(0)=\mu$ such that $w(s)>0$ for all $s>0$ and $\lim _{s \rightarrow \infty} w(s)=\beta^{1 / \alpha}$.

Proof. - We return to the context of the contraction mapping argument (proof of Theorem 2.5) as described at the start of the proof of Proposition 4.1. We claim that the set of data $\mu=w(0)$ and $v=w^{\prime}(T)$ which give rise to positive solutions converging to $\beta^{1 / \alpha}$ as $s \rightarrow \infty$ is open. Indeed, let $w$ be such a solution. Fix $T_{1}>\frac{1}{4(\gamma-1)}$ such that $H\left(T_{1}\right)<0$. If $z$ is another solution with $z(0)$ close to $\mu$ and $z^{\prime}(T)$ close to $v$, then $z(s)>0$ on $\left[0, T_{1}\right]$ and $H_{z, z^{\prime}}\left(T_{1}\right)<0$. Since $T_{1}>\frac{1}{4(\gamma-1)}$, it follows that $H_{z, z^{\prime}}^{\prime}(s) \leqslant 0$, and thus $H_{z, z^{\prime}}(s)<0$, for all $s \geqslant T_{1}$. This implies that $z(s) \neq 0$ for all $s \geqslant T_{1}$ and that $\lim _{s \rightarrow \infty} z(s)=\beta^{1 / \alpha}$. This proves the claim.

The result now follows by applying the claim to the solution identically equal to $\beta^{1 / \alpha}$.

THEOREM 4.4. - Assume $N>2$ and $\frac{2}{N-2}<\alpha<\frac{4}{N-2}$. There exists $\varepsilon>0$ such that for every $\mu, \beta^{1 / \alpha}<\mu<(1+\varepsilon) \beta^{1 / \alpha}$, there is a solution $w$ of $(1.12)$ with $w(0)=\mu$ such that $w(s)>0$ for all $s>0$ and $\lim _{s \rightarrow \infty} w(s)=0$.

Proof. - We again use the context of the contraction mapping argument of Theorem 2.5 as specifically described in the proof of Proposition 4.1, and we use the same $\varepsilon$ as in Corollary 4.3. For a fixed $\mu$, with $\beta^{1 / \alpha}<\mu<(1+\varepsilon) \beta^{1 / \alpha}$, the set of $v$ for which the solution with $w(0)=\mu$ and $w^{\prime}(T)=v$ is of the type described in Corollary 4.3 is open. Likewise, for a fixed value of $\mu>\beta^{1 / \alpha}$, the set of $v=w^{\prime}(T)$ which give rise to solutions becoming negative at some point is clearly open, and non-empty by Propostion 4.1. Thus, there exists a solution with $w(0)=\mu$ which is of neither of these two types: it must be positive but not converge to $\beta^{1 / \alpha}$. By Propositions 3.2 and 3.3, it must converge to 0 as $s \rightarrow \infty$.

We now turn to the critical and supercritical cases. We need to use the properties of solutions to the profile equation (1.6) with a specified initial value. More precisely, let $f_{\lambda}$ denote the solution of (1.6) such that $f_{\lambda}(0)=\lambda$ and $f_{\lambda}^{\prime}(0)=0$. We refer the reader to Section 3 and Theorem 5 of [4] for the facts about such solutions which we use below. In particular, the limit

$$
L(\lambda) \equiv \lim _{r \rightarrow \infty} r^{2 / \alpha} f_{\lambda}(r)
$$

exists and is a locally Lipschitz function of $\lambda \in \mathbb{R}$. Moreover, given any $\Lambda>0$, there exists $M=M(\Lambda)>0$ such that for all $\lambda \in[-\Lambda, \Lambda]$ and $r \geqslant 0$,

$$
\begin{aligned}
& (1+r)^{2 / \alpha}\left|f_{\lambda}(r)\right| \leqslant M, \\
& (1+r)^{2 / \alpha+1}\left|f_{\lambda}^{\prime}(r)\right| \leqslant M .
\end{aligned}
$$

While it is not explicitly stated, the proof of Proposition 3.1 in [4] shows that $M=M(\Lambda)$ can be taken arbitrarily small if $\Lambda>0$ is sufficiently small. Since $\alpha \geqslant \frac{4}{N-2}$, Theorem 5 in [4] tells us that if $\lambda>0$, then $f_{\lambda}(r)>0, \forall r>0$ and $L(\lambda)>0$. It follows in particular that for small $\lambda>0,0<f_{\lambda}(r)<V(r)$ for all $r>0$, where $V$ is the singular stationary solution given by (1.2), considered as a function of $r=|x|$. 
We next set $w_{\lambda}(s)=s^{-1 / \alpha} f_{\lambda}(1 / \sqrt{s})$, and so $w_{\lambda}$ and $f_{\lambda}$ are related by formula (1.9). Each $w_{\lambda}$ is a solution of (1.12) with $w_{\lambda}(0)=L(\lambda)$. Also, $w_{\lambda}(s)>0, \forall s \geqslant 0, \forall \lambda>0$ and $\lim _{s \rightarrow \infty} w_{\lambda}(s)=0$. Since the constant solution of (1.12) identically equal to $\beta^{1 / \alpha}$ corresponds under (1.9) to the singular solution $V$, which as a function of $r>0$ is also a solution of (1.6), it follows that if $\lambda>0$ is sufficiently small, then $w_{\lambda}(s)<\beta^{1 / \alpha}, \forall s \geqslant 0$. The estimates (4.1) and (4.2) translate for the $w_{\lambda}$ as

$$
\begin{aligned}
& w_{\lambda}(s) \leqslant \frac{M}{(1+s)^{1 / \alpha}}, \\
& s\left|w_{\lambda}^{\prime}(s)\right| \leqslant \frac{M}{(1+s)^{1 / \alpha}},
\end{aligned}
$$

for all $s \geqslant 0$ and all $\lambda \in[-\Lambda, \Lambda]$, where perhaps a different value of $M=M(\Lambda)$ is used. It follows from Remark 2.8 that for any fixed $\Lambda>0$, the collection of solutions, $w_{\lambda}, \lambda \in[-\Lambda, \Lambda]$, can all be obtained from Theorem 2.5 with the same values of $R, M$ and $T_{0}$, and also with $M$ replaced by $M+1$. This allows us to apply the conclusion of Proposition 2.7 for all such solutions on one common interval $[0, T]$.

LEMMA 4.5. - Suppose $\alpha \geqslant \frac{4}{N-2}$. If $0<\lambda_{1}<\lambda_{2}$ are such that $w_{\lambda_{1}}$ and $w_{\lambda_{2}}$ have a different number of intersections with the constant solution $\beta^{1 / \alpha}$, then there exists $\lambda \in\left[\lambda_{1}, \lambda_{2}\right]$ such that $w_{\lambda}(0)=\beta^{1 / \alpha}$.

Proof. - Suppose the conclusion is false. Without loss of generality, we assume that $w_{\lambda}(0) \leqslant \beta^{1 / \alpha}-\varepsilon$ for all $\lambda \in\left[\lambda_{1}, \lambda_{2}\right]$. Let $T>0$. The integral equation (2.5) implies that for $s \in[0, T]$ and $\lambda \in\left[\lambda_{1}, \lambda_{2}\right]$,

$$
w_{\lambda}(s) \leqslant \beta^{1 / \alpha}-\varepsilon+T^{2} K_{1}(T)\left|w_{\lambda}^{\prime}(T)\right|+T K_{2}(T) D M,
$$

where $K_{1}(T)$ and $K_{2}(T)$ are as in the proof of Proposition 2.5 , and $D=\max _{x \in[0, M]}\left|g^{\prime}(x)\right|$. It follows from (4.4) that one can choose $T>0$ small enough so that

$$
T^{2} K_{1}(T)\left|w_{\lambda}^{\prime}(T)\right|+T K_{2}(T) D M \leqslant \frac{\varepsilon}{2},
$$

and so $w_{\lambda}(s) \leqslant \beta^{1 / \alpha}-\frac{\varepsilon}{2}$ for all $s \in[0, T]$ and $\lambda \in\left[\lambda_{1}, \lambda_{2}\right]$. Thus, no intersections with the constant solution can occur on the interval $[0, T]$. By the estimates (4.3) and (4.4), no intersections with the constant solution $\beta^{1 / \alpha}$ can occur for $s$ sufficiently large, uniformly for $\lambda \in\left[\lambda_{1}, \lambda_{2}\right]$. Since no intersections can be introduced at some fixed, finite $s>0$, it must be that the $w_{\lambda}$, for $\lambda \in\left[\lambda_{1}, \lambda_{2}\right]$ all have the same number of intersections with the constant solution $\beta^{1 / \alpha}$. This contradiction proves the lemma.

LEMMA 4.6. - Suppose $\alpha \geqslant \frac{4}{N-2}$ and let $k>0$ be an integer. Let $\lambda_{0}>0$ be such that $w_{\lambda_{0}}(0)=\beta^{1 / \alpha}$ and $w_{\lambda_{0}}$ has precisely $k$ intersections with the constant solution $\beta^{1 / \alpha}$, including the one at $s=0$. It follows that there exists $\varepsilon>0$ such that if $\lambda \in$ $\left(\lambda_{0}-\varepsilon, \lambda_{0}+\varepsilon\right)$, then $w_{\lambda}$ has either exactly $k$ or exactly $k-1$ intersections with the constant solution $\beta^{1 / \alpha}$.

Proof. - By the second statement in Proposition 2.7, there exists $T_{1}>0$ such that each solution $w_{\lambda}, \lambda \in\left[\lambda_{0} / 2,2 \lambda_{0}\right]$ has at most one intersection with the constant solution $\beta^{1 / \alpha}$ 
on the interval $\left[0, T_{1}\right]$. Since $w_{\lambda_{0}}$ indeed has an intersection with the constant solution on $\left[0, T_{1}\right]$, i.e. at $s=0$, it follows that the other solutions have either the same number or one less intersection on [0, $\left.T_{1}\right]$. Next, by (4.3) and (4.4) there exists $T_{2}>0$ such that the solutions $w_{\lambda}, \lambda \in\left[\lambda_{0} / 2,2 \lambda_{0}\right]$ have no intersections with the constant solution for $s \geqslant T_{2}$. Note that that $w_{\lambda_{0}}\left(T_{1}\right) \neq \beta^{1 / \alpha}$ and $w_{\lambda_{0}}\left(T_{2}\right) \neq \beta^{1 / \alpha}$. The result follows since we can choose $\varepsilon>0$ so that all the solutions $w_{\lambda}, \lambda \in\left[\lambda_{0}-\varepsilon, \lambda_{0}+\varepsilon\right]$ have the same number of intersections with the constant solution on the interval $\left[T_{1}, T_{2}\right]$.

Lemma 4.7. - Suppose $\alpha \geqslant \frac{4}{N-2}$ and let $k$ be a positive integer. If $\lambda_{1}>0$ and $\lambda_{2}>0$ are such that $w_{\lambda_{1}}(0)=w_{\lambda_{2}}(0)=\beta^{1 / \alpha}$ and that $w_{\lambda_{1}}$ and $w_{\lambda_{2}}$ have respectively at most $k$ and at least $k+2$ intersections with the constant solution $\beta^{1 / \alpha}$ (including the one at $s=0)$, then there exists $\lambda$ between $\lambda_{1}$ and $\lambda_{2}$ such that $w_{\lambda}(0)=\beta^{1 / \alpha}$ and $w_{\lambda}$ has exactly $k+1$ intersections with the constant solution $\beta^{1 / \alpha}$.

Proof. - (We may assume that $\lambda_{1}<\lambda_{2}$.) We claim first that there exists $\lambda \in\left(\lambda_{1}, \lambda_{2}\right)$ such that $w_{\lambda}(0)=\beta^{1 / \alpha}$. Suppose not. It follows from Lemma 4.5 that all the $w_{\lambda}$, $\lambda \in\left(\lambda_{1}, \lambda_{2}\right)$ have the same number of intersections with the constant solution. But this is impossible by Lemma 4.6: for $\lambda$ close to $\lambda_{1}, w_{\lambda}$ can have at most $k$ intersections with the constant solution, and for $\lambda$ close to $\lambda_{2}$, the $w_{\lambda}$ must have at least $k+1$ such intersections. This proves the claim.

If none of the $w_{\lambda}$ with $\lambda \in\left(\lambda_{1}, \lambda_{2}\right)$ and $w_{\lambda}(0)=\beta^{1 / \alpha}$ have precisely $k+1$ intersections with the constant solution, then by choosing an appropriate subinterval, we are reduced to the previous situation, with $w_{\lambda}(0) \neq \beta^{1 / \alpha}$ for all $\lambda \in\left(\lambda_{1}, \lambda_{2}\right)$.

LEMmA 4.8. - Suppose $\alpha \geqslant \frac{4}{N-2}$ and let $k$ be a positive integer. If $\lambda_{1}>0$ and $\lambda_{2}>0$ are such that $w_{\lambda_{1}}(0)=w_{\lambda_{2}}(0)=\beta^{1 / \alpha}$ and that $w_{\lambda_{1}}$ and $w_{\lambda_{2}}$ have respectively exactly $k$ and $k+1$ intersections with the constant solution $\beta^{1 / \alpha}$ (including the one at $s=0$ ), then there exists $\lambda$ between $\lambda_{1}$ and $\lambda_{2}$ such that $w_{\lambda}(0) \neq \beta^{1 / \alpha}$ and $w_{\lambda}$ has exactly $k$ intersections with the constant solution.

Proof. - As above, we suppose that $\lambda_{1}<\lambda_{2}$, and we argue by contradiction. Suppose that $w_{\lambda}(0)=\beta^{1 / \alpha}, \forall \lambda \in\left[\lambda_{1}, \lambda_{2}\right]$. By the second statement in Proposition 2.7, there exists $T_{1}>0$ such that these $w_{\lambda}$ all have the same number of intersections with the constant solution on $\left[0, T_{1}\right]$. Indeed, these intersections all take place at $s=0$. The estimates (4.1) and (4.2) and continuous dependence easily imply that the $w_{\lambda}$ all have the same number of intersections with the constant solution on $[0, \infty)$. This contradiction proves that there exists $\lambda$ between $\lambda_{1}$ and $\lambda_{2}$ such that $w_{\lambda}(0) \neq \beta^{1 / \alpha}$. If it happens that $w_{\lambda}(0) \neq \beta^{1 / \alpha}$ for all $\lambda \in\left[\lambda_{1}, \lambda_{2}\right]$, then it follows from Lemmas 4.5 and 4.6 that the $w_{\lambda}$ must all have exactly $k$ intersections with the constant solution. In fact, we can always reduce to this case by replacing $\lambda_{1}$ and $\lambda_{2}$ respectively by $\lambda_{1}^{\prime}$ and $\lambda_{2}^{\prime}$, where

$$
\begin{aligned}
& \lambda_{1}^{\prime}=\sup \left\{\lambda \in\left[\lambda_{1}, \lambda_{2}\right]:\right. w_{\lambda}(0)=\beta^{1 / \alpha} \text { and } w_{\lambda} \text { has exactly } k \text { intersections with } \\
&\text { the constant solution } \left.\beta^{1 / \alpha}\right\}, \\
& \lambda_{2}^{\prime}=\inf \left\{\lambda \in\left[\lambda_{1}^{\prime}, \lambda_{2}\right]:\right. w_{\lambda}(0)=\beta^{1 / \alpha} \text { and } w_{\lambda} \text { has exactly }(k+1) \text { intersections with } \\
&\text { the constant solution } \left.\beta^{1 / \alpha}\right\} .
\end{aligned}
$$

This proves the lemma. 
Proof of Theorem 1.4. - By the (proof of) Proposition 3.8 in [4], rescaled versions of the $f_{\lambda}$ given by $\lambda^{-1} f_{\lambda}\left(\lambda^{-\alpha / 2} r\right)$ converge uniformly on compact subsets of $[0, \infty)$ as $\lambda \rightarrow \infty$ to the regular stationary solution of (1.1) $v$, which satisfies the initial value problem:

$$
\begin{aligned}
& v^{\prime \prime}(r)+\frac{N-1}{r} v^{\prime}(r)+|v(r)|^{\alpha} v(r)=0, \\
& v(0)=1, \quad v^{\prime}(0)=0 .
\end{aligned}
$$

Joseph and Lundgren [5, Sections VII and VIII] have shown that if $\frac{4}{N-2}<\alpha<\alpha^{*}$, then $v$ has infinitely many intersections with the singular stationary solution of (1.1), $V(r)=\beta^{1 / \alpha} r^{-2 / \alpha}$. It follows that for large $\lambda>0, f_{\lambda}$ has an arbitrarily large number of intersections with the singular solution $V$. Thus, for large $\lambda>0, w_{\lambda}$ has an arbitrarily large number of intersections with the constant solution $\beta^{1 / \alpha}$ of (1.12). Since for $\lambda>0$ small enough, $w_{\lambda}$ has no intersection with the constant solution, it follows from the Lemmas 4.5, 4.6, and 4.7 that there exists a sequence $\lambda_{k}, k=1,2,3, \ldots$, such that $\lambda_{k}<$ $\lambda_{k+1}, w_{\lambda_{k}}(0)=\beta^{1 / \alpha}$, and $w_{\lambda_{k}}$ has precisely $k$ intersections with the constant solution. It then follows from Lemma 4.8 that there exists a second sequence $\lambda_{k}^{\prime}, k=1,2,3, \ldots$, such that $\lambda_{k}<\lambda_{k}^{\prime}<\lambda_{k+1}, w_{\lambda_{k}^{\prime}}(0) \neq \beta^{1 / \alpha}$, and $w_{\lambda_{k}^{\prime}}$ has precisely $k$ intersections with the constant solution $\beta^{1 / \alpha}$. Since the parity (even or odd) of the number of intersections that $w_{\lambda_{k}^{\prime}}$ can have with the constant solution depends on whether $w_{\lambda_{k}^{\prime}}(0)$ is greater than or less than $\beta^{1 / \alpha}$, it follows that the $w_{\lambda_{k}^{\prime}}(0)$ are alternately greater than or less than $\beta^{1 / \alpha}$. This implies that $L(\lambda)$ is greater than (respectively less than) $\beta^{1 / \alpha}$ on an infinite set of disjoint open intervals separated by points where $L(\lambda)$ equals $\beta^{1 / \alpha}$.

This proves Theorem 1.4.

Proof of Theorem 1.3. - The proof of Theorem 1.3 uses the same reasoning as in the proof of Theorem 1.4. The only difference is that the singular stationary solution $V$ and the regular stationary solution $v$, which is known explicitly in this case, intersect each other precisely twice.

\section{Acknowledgement}

The authors are grateful to M. Fila for helpful remarks during the preparation of this paper.

\section{REFERENCES}

[1] T. Cazenave, F.B. Weissler, Asymptotically self-similar global solutions of the nonlinear Schrödinger and heat equations, Math. Z. 228 (1998) 83-120.

[2] C. Dohmen, M. Hirose, Structure of positive radial solutions to the Haraux-Weissler equation, Nonlinear Anal. TMA 33 (1998) 51-69.

[3] V.A. Galaktionov, J.L. Vazquez, Continuation of blowup solutions of nonlinear heat equations in several space dimensions, Comm. Pure Appl. Math. 50 (1997) 1-67.

[4] A. Haraux, F.B. Weissler, Non-uniqueness for a semilinear initial value problem, Indiana Univ. Math. J. 31 (1982) 167-189. 
[5] D.D. Joseph, T.S. Lundgren, Quasilinear Dirichlet problems driven by positive sources, Arch. Rat. Mech. Anal. 49 (1973) 241-269.

[6] L.A. Peletier, D. Terman, F.B. Weissler, On the equation $\Delta u+\frac{1}{2} x \cdot \nabla u+f(u)$, Arch. Rat. Mech. Anal. 94 (1986) 83-99.

[7] J.L. Vazquez, Domain of existence and blowup for the exponential reaction-diffusion equation, Indiana Univ. Math. J. 48 (1999) 677-709.

[8] F.B. Weissler, Asymptotic analysis of an ordinary differential equation and non-uniqueness for a semilinear partial differential equation, Arch. Rat. Mech. Anal. 91 (1986) 231-245.

[9] F.B. Weissler, $L^{p}$-energy and blow-up for a semilinear heat equation, Proc. Symp. Pure Math. Part II 45 (1986) 545-551.

[10] E. Yanagida, Uniqueness of rapidly decaying solutions of the Haraux-Weissler equation, J. Differential Equations 127 (1996) 561-570. 